\title{
Risk Factors for and Long-term Outcomes of Anastomotic Leakage After Colorectal Cancer Surgery: Reply
}

\author{
Mark A. Boccola Petra G. Buettner • \\ Warren Matthew Rozen · Yik-Hong Ho
}

Published online: 7 April 2011

(c) Société Internationale de Chirurgie 2011

We greatly appreciate Dr. Akiyoshi and colleagues' interest in our manuscript titled Risk Factors and Outcomes for Anastomotic Leakage in Colorectal Surgery: A Singleinstitution Analysis of 1,576 Patients [1]. The authors explain that their primary concern was the validity of our multivariate analysis based on a perceived disagreement of numbers. Their comment, "elective procedures of bowel resection in combination with $(N=1,430)$ or without $(N=38)$ gynecological procedures were subjected to multivariate analysis. However, Table 1 showed that 266 of 1,576 patients underwent emergency operation, and that is, elective operation was performed in 1,310 patients" expresses concerns that a number of elective cases reported in Table 1 have been excluded from the multivariate analysis; however, this is not the case. Unfortunately, it seems they have misread the data in Table 2 by swapping number of elective operations in combination with gynecological procedures $N=38$ with all other operations $N=1,430$, not the other way around, and this has led to their erroneous conclusion of data error. We accept that the category of "all other operations" may be unclear, as it does in fact include emergency and elective operations. However, as noted in the footnote to that table, all data have been adjusted for confounding in regard to this variable, among others.

Akiyoshi et al. justly raise a concern in relation to use of drain tubes. They state that "use of a drain should be subjected to multivariate analysis, because the authors showed that abdominal drain via laparoscopic port was a significant factor associated with anastomotic leakage by

M. A. Boccola · P. G. Buettner · W. M. Rozen $(\bowtie) \cdot$ Y.-H. Ho Discipline of Surgery, James Cook University,

Townsville, QLD, Australia

e-mail: warrenrozen@hotmail.com univariate analysis." In fact, our article used bivariate analysis to show that anastomotic leak was more likely after abdominal drain via a stab wound, and we did perform a multivariate analysis for this variable. However it did not achieve statistical significance. Level 1 evidence has previously shown that using a drain does not preclude anastomotic leak or minimize the severity of related complications in colorectal anastomoses [2, 3], which was contrary to the finding of our study.

Lastly, the authors requested consideration of a multivariate survival analysis (Cox model) for anastomotic leak. Anastomotic leakage remained a significant prognostic factor in multivariable survival analyses. General anastomotic leakage $(p<0.001)$ negatively influenced overall survival when adjusted for age, gender, TNM stage, histological grading, tumor site, obstruction and perforation, venous invasion, type of surgical procedure, adjuvant therapy, emergency operation, serious medical complications, and serious surgical complications. Local anastomotic leakage ( $p=0.037)$ remained statistically significant for cancerrelated mortality when adjusted for the same factors, whereas general anastomotic leakage ( $p=0.049)$ remained significant within this Cox model for disease-free survival.

\section{References}

1. Boccola MA, Buettner PG, Rozen WM et al (2011) Risk factors and outcomes for anastomotic leakage in colorectal surgery: a single-institution analysis of 1,576 patients. World J Surg 35:186-195

2. Urbach DR, Kennedy ED, Cohen MM (1999) Colon and rectal anastomoses do not require routine drainage: a systematic review and meta-analysis. Ann Surg 229:174-180

3. Jesus EC, Karliczek A, Matos D et al. (2004) Prophylactic anastomotic drainage for colorectal surgery. Cochrane Database Syst Rev (4):CD002100 